\title{
Approaches for Hyperspectral Image Classification- Detailed Review
}

\author{
Kushalatha M R, Prasantha H S, Beena R. Shetty
}

\begin{abstract}
Abstarct: Hyperspectral Image (HSI) processing is the new advancement in image / signal processing field. The growth over the years is appreciable. The main reason behind the successful growth of the Hyperspectral imaging field is due to the enormous amount of spectral and spatial information that the imagery contains. The spectral band that the HSI which contains is also more in number. When an image is captured through the HSI cameras, it contains around 200-250 images of the same scene. Nowadays HSI is used extensively in the fields of environmental monitoring, Crop-Field monitoring, Classification, Identification, Remote sensing applications, Surveillance etc. The spectral and spatial information content present in Hyperspectral images are with high resolutions. Hyperspectral imaging has shown significant growth and widely used in most of the remote sensing applications due to its presence of information of a scene over hundreds of contiguous bands In. Hyperspectral Image Classification of materials is the critical application of HSI using Hyperspectral sensors. It collects hundreds of spectrum channels, where each channel consists of a sharp point of Electromagnetic Spectrum. The paper mainly focuses on Deep Learning techniques such as Convolutional Neural Network (CNN), Artificial Neural Network (ANN), and Support Vector machines (SVM), K-Nearest Neighbour (KNN) for the accuracy in classification. Finally in the summary the current state-of-the-art scheme, a critical discussion after reviewing the research work by other professionals and organizing it into review-based paper, also implying about the present status on classification accuracy using neural networks is carried out.
\end{abstract}

Keywords: Hyperspectral imaging (HSI), Supervised and Unsupervised Classification, Semi - Supervised Classification, Neural Network, Classification accuracy

\section{INTRODUCTION}

Hyperspectral imaging is considered to be a booming research area in remote sensing where for the same spatial area, an imaging spectrometer collects hundreds (around 100 to 200 bands) of bands of images at different wavelengths.

Manuscript received on July 29, 2021.

Revised Manuscript received on August 13, 2021.

Manuscript published on September. 30, 2021.

* Correspondence Author

Kushalatha M R*, Assistant Professor, Department of Electronics and communication Engineering, Nitte Meenakshi Institute of Technology, Bangalore, India.

Prasantha H S, Professor, Department of Electronics and Communication Engineering, Nitte Meenakshi Institute of Technology (Affiliated to VTU, Belgaum), Bangalore

Beena R. Shetty, Assistant Professor, Department of Electronics and Communication in Nitte Meenakshi Institute of Technology, Bangalore

(C) The Authors. Published by Blue Eyes Intelligence Engineering and Sciences Publication (BEIESP). This is an open access article under the CC BY-NC-ND license (http://creativecommons.org/licenses/by-nc-nd/4.0/)
Hyperspectral imaging helps to collect the radiance or intensity level for each pixel in the image of a scene with substantial number of narrow bands available, detects objects, finds the materials and also helps in detecting various processes. Basically three types of sensors are widely used in Hyperspectral imaging: (i) The push broom scanners and the related whisk broom scanners:, helps in image scanning (ii) The band sequential scanner helps in spectral scanning, where images of an area pertaining to different wavelengths are captured (iii) The snapshot Hyperspectral imaging is the third variation of sensors where an staring array is used to create an image in a given instant.The researches carried out in last few decades extracted a lot amount of information with respect to spatial, temporal and spectral resolutions. The HSIs carry a generous amount of spectral information, which introduced many newer applications, new technological challenges and advancement in newer domains of research [1]. The high spectral resolution made the HSI to be used in extraction of subtle objects, materials with and are widely used in applications like remote sensing, quantification [2], identification, surveillance [3] and in precision urban agriculture [4].The first Indian Imaging Satellite by (HySIS) was launched by ISRO on $29^{\text {th }}$ November which captured HSI images and used for earth observations, applications related to forestry, geology, assessment of coastal zones, agriculture and studies related to environment. The HySIS imaging combines digital imaging and spectrometry where the images acquired is of high contrast. The satellite not only captures solids and liquids but also detects them. The visible region and near IR are used to detect the images with around 55 colour bands. Currently NASA is also working on Hyperspectral Image classification techniques. The worked carried out by capturing the Hyperspectral images by NASA in the field of agriculture has better classification characteristics, better modelling and mapping of the Hyperspectral data. The classification efficiency observed is in the range of 8895.17\%. Very limited organisations are working on HSI data, The Image Processing Laboratory -University of Valencia, Spain is one among them. The current area is exploring the machine learning, image processing and signal processing using machine learning platforms. The current work focuses on retrieval of biophysical parameter, inversion model in image classification and segmentation, target detection $n$ separation of source for Hyperspectral images. The process of assigning the individual pixels to a definite group of classification classes (Trained or Untrained depending on Supervised or Unsupervised classification) is a tedious process in classification of images.

\section{Published By:}

Blue Eyes Intelligence Engineering and Sciences Publication

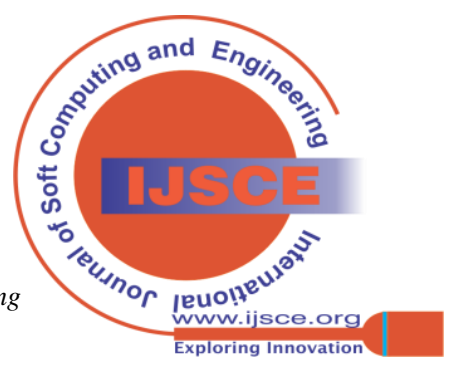


The training samples available in a classification approach can be divided into mainly 2 categories, i.e., Supervised and Unsupervised classifiers. In the supervised approach the classification procedure is based on (classification of input data) already available samples known as training samples where in unsupervised doesn't have such available samples for classification purpose. The HSI classification has taken a large amount of advances with respect to its classification efficiency; it still poses problems with respect to high dimensionality, unbalanced and limited number of training samples available, mixing of pixels and training samples [5]. Second problem is that; the classification efficiency reduces when less number of samples is taken. The third problem arises with the curse of increment in dimensions of data and high redundancy between the features, which make the classification in its analysis stage. A large degree of literature surveys has been conducted as to overcome these problems, and to conduct Hyperspectral image classification in an effective way.Convolutional Neural Networks, Maximum likelihood (ML) methods, neural networks architectures [6], support vector machine (SVM) [7], Bayesian approach [8] as well as Kernel methods [9] are the proposed methods used in the recent years with respect to classification of Hyperspectral images. Based on the methods of utilisation of the available training samples, image classification process is categorized as Supervised, Unsupervised and Semi-Supervised Hyperspectral Image classification.

\section{SURVEY CARRIED OUT}

Several scientists and researchers from different part of the world have proposed and implemented classification techniques for monitoring the crop condition and variety using different approaches of image processing, in the recent times. A detailed review of pre-processing techniques, identification of the algorithm, feature extraction techniques used, classification techniques and the tool used for the various types of images are listed here. Lan et al. (2019) proposed a newer method on the K-sparse de-noising autoencoder and HSI characteristics of unique with restricted spectral information. The method proposed a solution for considerable dissimilarities seen in the spatial content of the same pixel within the neighbouring region. The increase in efficiency of KDAE indicated that the spectral-limited spatial features are advantageous for classification. The main drawback was in the system to resolve the difficulty of significant dissimilarities in the SI of the same pixel within the neighbouring region [10]. Hasan et al. (2019) compared the different techniques like Support Vector Machine (SVM), Artificial Neural Network (ANN) and Convolution Neural Network (CNN). The tools used were Confusion matrices, Traditional statistical method, Structural Risk Management. The thermal infrared $(2.5$ to $14.0 \mu \mathrm{m})$ Hyperspectral spectrum range was used for classification of thirteen different plant species. The overall accuracy obtained by CNN, ANN and SVM is 99\%, 94\% and 91\%, respectively [9]. Qureshi et al. (2019) described the Hyperspectral document image processing methods and its applications. Research field focused on overcoming the increased image speed and on reducing the camera cost HSI. The methodology helped to solve problems related to image analysis, including document or ink aging, signature

Published By:

Blue Eyes Intelligence Engineering and Sciences Publication

(C) Copyright: All rights reserved. extraction, and retrieval of information. The drawback of this document imaging was relative high cost. The conclusion and future study recommended having small and portable Hyperspectral document imaging, which includes CCD sensors, spectral filters, and illumination [10]. Zhao et al. (2019) presented a new algorithm of multiple Convolutional layers fusion which extracts from course to fine scales along with layers which adopted two fusion networks fully CNN and side output decision fusion network (SODFN). The data collected from different layers of Convolutional layers for HSI classification was taken. This fusion approach showed improvement in classification performance as well as generation capability compared to other fusion networks [11]. Ergul et al. (2019) proved that HCK boost for HSI classification is a standard method which does not involve complicated optimization while comparing with a minimum cost method. The future scope showed that there can be improvement in the spatial features [12]. Pan et al. (2018) presented the combined active learning (AL) and semi-supervised methods for classification of Hyperspectral Images. The method was tested with different labelled samples and superiority in classification maps in training samples. The classification approach provides an effective pseudo-labelling scheme, which makes full use of human labelling efforts. The drawback was this method can be applicable only for labelled samples [13]. Shwetank et al. (2018) represented a face image classification technique for Hyperspectral datasets. The supervised classification and Hyperspectral face images with Maximum Likelihood approaches were used for classification of face images and maximum response were achieved. The proposed technique would make it potential to formulate an advance and robust biometric authentication system based on techniques of image processing rather than instruments in the range of Hyperspectral [14]. Researcher Shao et al. (2018) came up with a model based on spatial and class structure regularized sparse representation (SCSSR) for HSI classification. The method incorporated SI via the Laplacian regularization with similar coefficients. The method showed improvement in the SR graph performance with spatial neighbourhood information and in the performance of a classifier. The drawback was that this method cannot be applicable on a sequential data, which is the future direction to improve the algorithm based on GAN to solve semi-supervised HSI classification [15]. Guo et al. (2018) proposed Recurrent Neural Network (RNN) for HSI classification. The classification model was used to solve the steady-state model and the method gained high classification performance $\mathrm{n}$ terms of efficiency. By taking $10 \%$ of training samples it achieved better accuracy compared with other SVM classifier and Auto encoder model [16]. Pan et al. (2017) proposed a Multi-Grained Network (Mug Net) in HSI classification, which mainly targets at limited models.

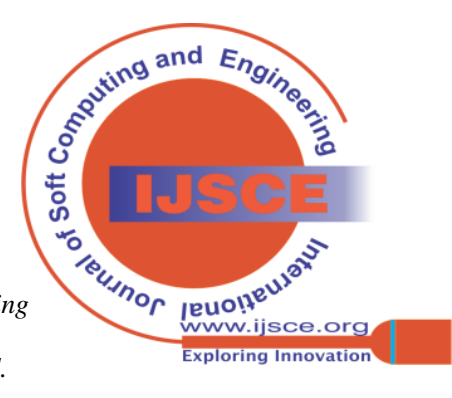


The method was the combination of three novel strategies, spectral relationship, convolution kernels, and spatial correlation. The output showed better classification results in HSI classification even in the case of the smallscale training set. However, the end-to-end manner for enlightening the computational efficiency was not taken care in this method proposed [17]. Wang et al. (2017) proposed cone-based joint sparse model (C-JM). The method implemented simultaneous orthogonal matching pursuit (SOMP) algorithm, and it resulted in a more stable and higher classification than any other means. The proposed CJSM model can be collaboratively attended with the window adaptation approaches for improving the classification concert, but it only ensures sparsity, spatial coherence, or non-negativity, which is the main drawback. The method was preferred to exploit the non-linearity demonstration [18]. Haoet al. (2016) proposed a classification using two regularizes, i.e., interclass basis sharing and inter-class essential competition and focused on three parts such as sparse features calculation, classification, and dictionary learning. The research resulted in minor computational price and improvement in the discriminative capability in the dictionary. The application of the unsupervised learning model on the data was not discussed [19]. Gu and Liu (2016) proposed an operative S2MKL technique to succeed in the classification of the Hyperspectral data to solve the optimization tasks. Three typical Hyperspectral data with groups of many altered contexts were taken and recorded by different sensors. The outcomes were consistent which demonstrated that the proposed method attained enhanced accuracy than both RMKL and RBMKL methods and resolves grouping coefficients of MKL by boosting systems. The limitation of the study was that it was used for limited training samples [20]. Sowmya et al. (2016) proposed an algorithm for dimension reduction and classification of Indian Pines and Salinas-A datasets, using the techniques of band selection approach. The computational difficulty was the main concern while conserving the critical information in the HSI. The results showed that even with a 50\% discount in dimension, the precisions were well achieved from kernel-based to that of the real image [21].

\subsection{Unsupervised Classification}

The main challenge posed by the classification approaches in Hyperspectral imaging is high dimensionality. As to overcome these curse varieties of feature extraction techniques are extensively made use of in classification process. The prominent features are only selected as to reduce the problem of dimensionality instead of selecting the entire features in an image. In case of unsupervised techniques, the automatic selections of group of pixels which carry similar information like means, deviations are done. The concept is based on type of algorithms used. The advantage of this type of classification is, it does not require prior information about how to train the data is required. Principal Component Analysis (PCA) [22], Independent Component Analysis (ICA) [23] is some of the very famous classification techniques under Unsupervised Classification.

2.1.1. Principal Component Analysis (PCA): PCA is mainly used method in classification as it helps in dimensionality reduction. The dimensionality reduction is accomplished by reducing the number of variables while in the original dataset most of the information is still retained. The basis on which the PCA works is the existence of good correlation between Hyperspectral image bands. PCA mainly deals with the second order statistics and these statistics cannot describe the higher resolution data, which are mainly made use of in Hyperspectral imaging.

2.1.2. Independent Component Analysis (ICA): The failure of PCA to deal with higher order statistics is eliminated by ICA. The method reduces the problem that occurs due to high dimensionality to a greater extent. ICA is well suited for reduction in dimensionality, extraction of features, retrieval and preservation of second order statistics $[25,26]$.

\subsection{Supervised Classification}

The supervised classification takes the data which is labelled in prior as to train the classifier. The advantage of supervised with respect to the unsupervised is that it can be applied to those images which contains rich spectral information. Supervised classification approach can be used in many applications like urban vegetation growth monitoring, changes in the landscape efficient resource management skills and techniques. Many supervised classification techniques have been widely used which is application and user dependent. Maximum likelihood (ML), nearest neighbour classifier, decision trees, random forest, and support vector machines (SVMs), Spectral Angle Mapper (SAM) etc are some of the widely used techniques of classification of the given Hyperspectral imagery.

2.2.1. ML Classifier (MLC): The ML classifier works on the assumption that, each class in each band of the image has a normal distribution and chosen training samples have definite defined classes. The assumption is done on estimation of probability of calculation of a specific class. A probability threshold must be selected as to carry smooth classification process. In the next stage allocation of each of the pixels of taken image to a particular class defined is carried out. The process of classification stops if the estimated probability is found to be smaller than a threshold set. As to implement the ML classifier technique estimation of mean vectors and covariance matrix using training process to be done at the first instance [27]. The accuracy of classification is higher compared to most of the other classification approaches. The discrimination of land cover classes is not easy as Hyperspectral images are the images that differ with respect to multispectral images with respect to the hundreds of bands that they carry. As the ML classifier works only with the accurate selection of training samples, Hyperspectral images with the poorly represented training samples won't give expected efficiency of classification. It is better to go for alternative methods for a multiclass classification.

2.2.2. K- Nearest Neighbour Algorithm (KNN): The proposed method has the following steps. Initial step is to adapt a support vector machine (SVM) technique. The SVM helps to obtain classification probability maps which in turn help to reflect the probability with which each of the pixels of the HSI belong to the different classes of classification.

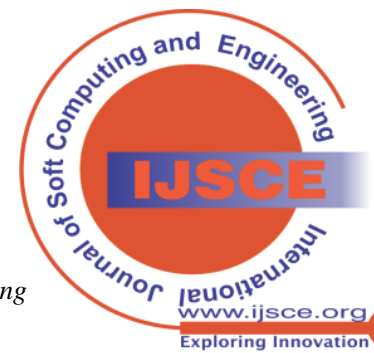


The filtering process of KNN includes matching and nonlocal averaging of the neighbourhood pixels. The method is proved to be a competitive method for classification as it doesn't make use of and optimization strategies. The KNN when applied on two widely used data sets proved that classification efficiency is better compared to the traditional methods. The KNN method's operating principle is based on a rule: majority voting rule, which presumes that all the neighbouring pixels are equally contributing to the classification process. Another important parameter used by KNN is Euclidian distance metric, which works as a distance metric when the data is homogeneous.

2.2.3. S pectral Angle Mapper (SAM): SAM is one of the types of supervised classification technique used for classification of Hyperspectral data. The method makes use of the information regarding the spectral angle of an image, thus obtains the classification very quickly. The method is very quick as it determines the reference spectra from its field measurements. The spectral angle measures the n-dimensional vector between the testing image and the reference spectrum with which the image is compared. The value of the angle determines the similarity and the difference between the image and the spectrum. If the angle is found to be larger, then the similarity is smaller and vice versa.

2.2.4. Support Vector Machine (SVM): SVM is a very strong and prominent classification approach, which makes use of Kernel functions and works on the basis of theory of optimization. SVM is proved to a better technique of classification in environments where the image is represented in more number of spectral bands and less number of training samples. Figure 1 shows a SVM model in multidimensional space which represents different classes in a Hyperplane. The process of iteration is incorporated as to generate the Hyperplane which reduces the error in classification. The maximum Marginal Hyperplane (MMH) is obtained by SVM by dividing the given datasets into variety of classes.
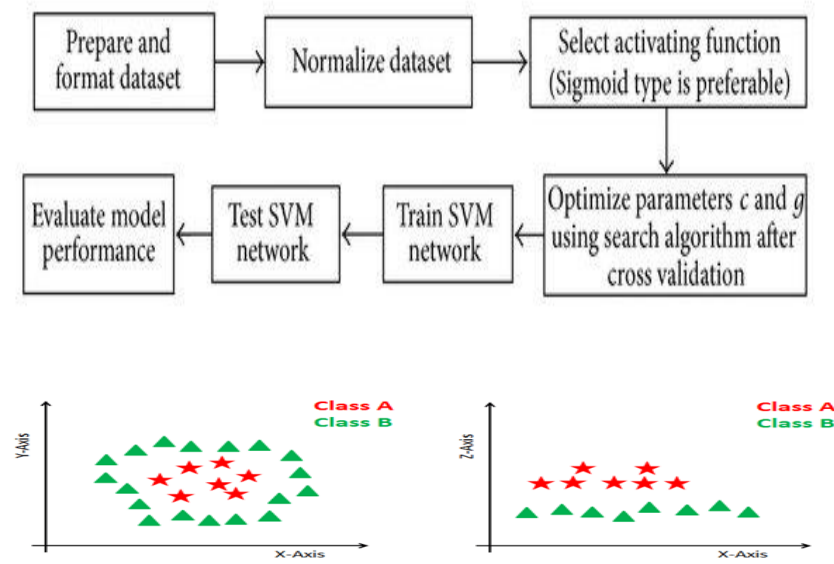

Figure1: General block diagram representation of SVM classifier and Representation of Maximum Marginal Hyperplane (MMH)

2.5. Random Forest Classifier (RFC): The random forest (RF) classifier is a supervised classification technique. The method is based on the concept of tree based classifiers. The individual tree is a pre- trained with a set of available training data. The input to the classifier is the data or images that is to be classified and the measure to be taken to feed

Published By:

Blue Eyes Intelligence Engineering and Sciences Publication

(C) Copyright: All rights reserved. this input data/images to each tree present in the forest. Each tree is involved in classification process and is known by the term "vote" for that class. The forest having the highest vote is selected by the forest for the classification process. The determination of split is important in the classification process which is accompanied by a process of searching through a subset which is random in nature and also is of variable in nature at each node in the tree. The Random forest classifier is extensively used due to its prominent characteristics like high accuracy and high speed of processing. In RFC care should be taken with respect to choosing the independence and correlation that actually affects the accuracy of the final cover map.

2.2.6. Spatial Spectral Classifier (SSC): The main disadvantage that the pixel - wise classifier method poses is difficulty in the discrimination of the classes due to its interclass spectral variability. The solution for the problem is making use of spatial dependency which in turn enhances the classification accuracy. Combining the spatial information into HSIC classification [29] the interclass classification information can be enhanced. This process of improvement motivates to study the spatial - spectral classification methods. The study of extraction of information involves and revolves around two entities, i.e., pixel and the label. The concept of neighbouring pixels and their spatial relations are the major studies that to be studied in evaluation of SSC.A relationship exists between pixel dependency and spatial dependency which in turn determines the level of correlation between the neighbouring pixels and labels. The following approaches are used for classification.

2.2.6.1. Structural Filtering: Concept of windowing techniques are used in this method as to extract the information related to the spatial content and their interrelationship in a Hyperspectral image. The approaches like spectral - spatial wavelet features, Gabor features and Weiner filtering methods are used.

2.2.6.2. Morphological Profile (MP): The spatial relationship between pixels using shape and size named by structuring element is used in investigating the spatial relationship between the pixels in Mathematical morphology. The different techniques in terms of nonlinear image processing that can be used in MP involve operations like dilation and erosion. The process of obtaining the information about contrast and size of the structures present in an image is termed as Granulometry. The MP of size ' $n$ ' can be obtained by the Granulometry process by opening the reconstruction and closing the reconstruction.

2.2.6.3. Random Field (RF): Markov Random Fields (MRFs) and Conditional Random Fields (CRFs) [30] are the extensively used methods in Random Field techniques is classification of HSI. In CRFs conditional probability is used for labelling of the data and optimal spatial information are used for favourable performance; wherein MRF estimates the parameters related to the classes independently from the parameters obtained from field. The estimated complexity in RF method of classification is found to be lesser.

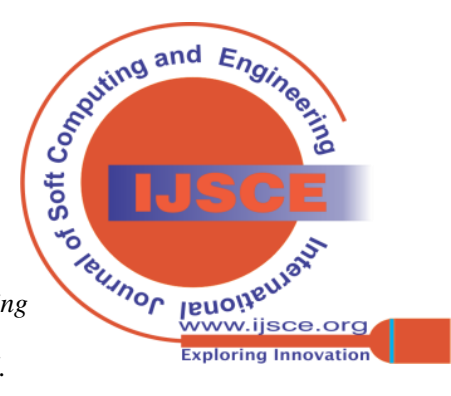


2.2.7. Sparse Representation Based Classification: In a sparsity-based algorithm initially a structured dictionary is formed, which contains a group of trained samples. Linear combining methods, sparse representation of the pixels are incorporated in this approach as to improve the performance of classification. Recovery of the sparse vector is the next stage which helps to label the classes for the given samples. The Non Local Weighted Joint Sparse Representation (NLW- JSRC) [30] method is a well-known method in sparse representation category; a method helps in removal of the invalid pixels. The classification approach proved that the discriminative dictionary resulted from the training samples can reduce the complexity in classification that occurs in classification.

2.2.8. Deep Learning (DL): The deep learning technique makes use of very deep neural networks, which tries to hierarchically learn deep features related to the Hyperspectral input data and are deeper than 3 layers [31]. In the first layer, the network is initialised through unsupervised training and later through a supervised manner. The low level features help to formulate the proper features in the beginning stage and proper features are used for classification of patterns at the end stage. Deep learning models results in more relevant features and are constant to the variations that takes place in the data at the input.

Spatial Dependency System

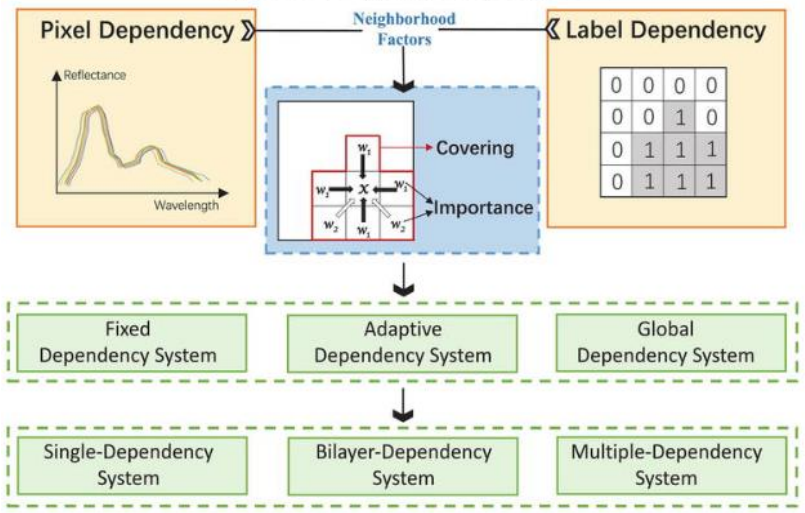

Figure 2: Block Diagram Representation of Spatial dependency system used in Spectral-Spatial Classification Process (Source Intechopen)

2.2.8.1. Deep Learning in HSI Classification: The DL is the type of unsupervised feature learning technique which makes use of a large amount of image dataset. Multiple layers or levels of information that exists between the data can be organised with the help of DL based techniques. The neural network based DL process becomes complex when Hyperspectral Imaging is taken into consideration. The deep learning models of Hyperspectral data consists of mainly 3 layers (i) Input Data Layer (ii) Construction of Deep Layer (iii) Process of Classification. The different approaches are Stacked Auto Encoder (SAE), Convolutional Neural Network (CNN) and Deep Belief Network (DBN). DBN is an unsupervised classification technique and trains one layer at a time .DBN performs unsupervised techniques on unlabelled samples first and he supervised on labelled samples at the end. The pre-trained DBN obtains the required information from the unlabelled samples. The technique fine tunes the small number of labelled samples. The factors related to dead or potential over tolerant in DBN are related to the decrement in the model source which in turn reduces the classification ability. As to overcome this problem the latent factors diversely to be done. The enhancement carried in the classification performance using the diversification of redundant factors of a given model has become trade research topic nowadays. Latent models are one of the important elements in machine learning classification technique. The DBN structure is as shown in Figure 3.

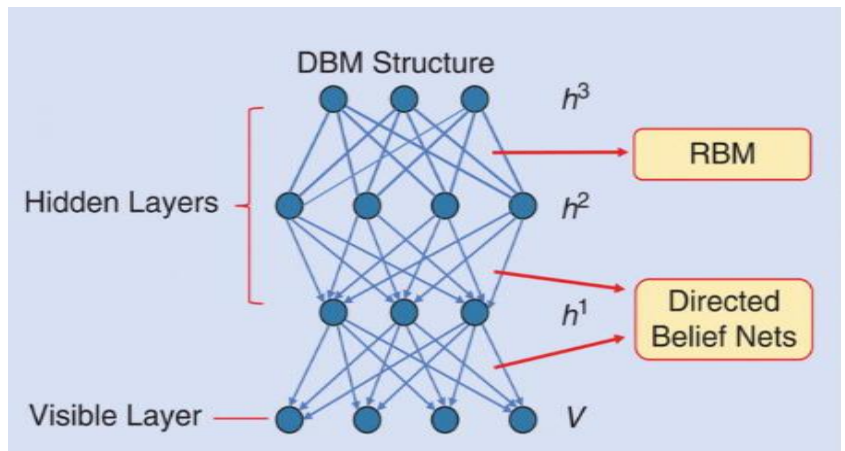

Figure 3: Structure of DBN (Source Inechopen)

The Diversified DBN (D- DBN) method used in classification contains two data sets namely Indian Pines and the University of Pavia scenes [32]. The spectral channels available in the Indian Pines data are 200 of 0.4 to $2.45 \mu \mathrm{m}$ range in visible and IR spectrum. The resolution in terms of its spatial content of these images was found to be is $20 \mathrm{~m} \times 20 \mathrm{~m}$. Among the 200 images around 20 images are discarded due to noise and water absorption. The University of Pavia contained around 115bands with $610 \times 340$ pixels and a possessed resolution of $1.3 \mathrm{~m} \times 1.3 \mathrm{~m}$ in terms of its spatial content. The classification and computational performance of the DBN can be enhanced by making a considerable modification in the pre- training of the samples and by fine-tuning of D-DBNs. The methods related to DBN classification produce very fast inference and helps in identifying the Hyperspectral images in a competitive manner. DBN based classification approach resulted in a good classification performance. A false colour image (3 bands) and the ground truth data are depicted in Figure 4 and 5 .

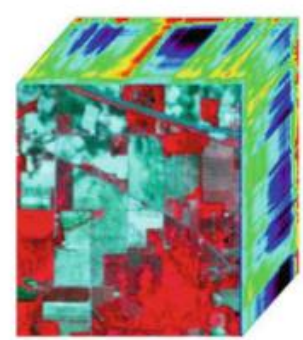

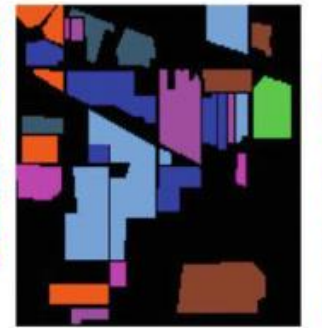

(b)

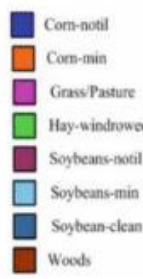

(c)
Figure 4: Indian Pines data set (a) False colour image (shown in 3 bands) (b) Ground Truth with 8 classes (c) Representation of mapping of colours

\section{Published By:}

Blue Eyes Intelligence Engineering and Sciences Publication (C) Copyright: All rights reserved.

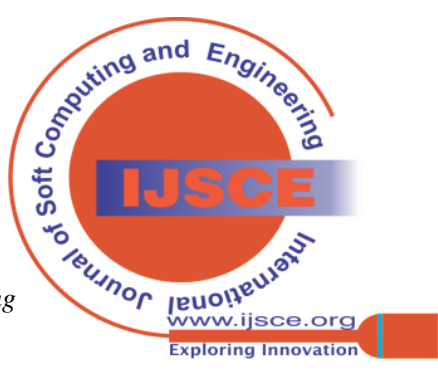




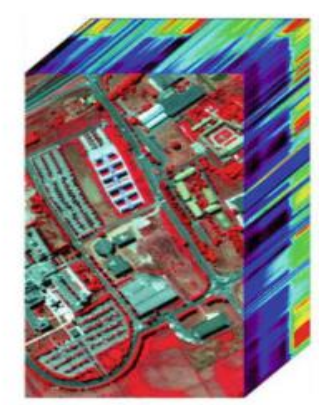

(a)

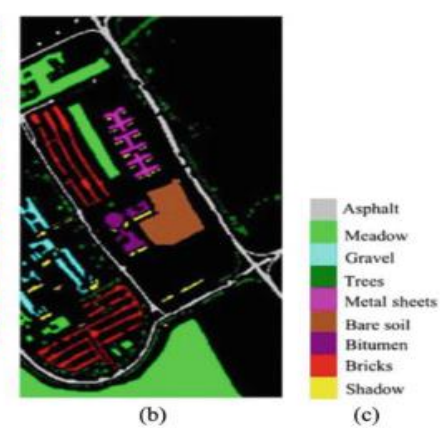

(c)
Figure 5: University of Pavia data set (a) False colour image (Shown in 3 bands) (b) Ground Truth with 9 classes (c) Representation of mapping of colours

2.2.8.2. Convolutional Neural Networks (CNN): The CNN is the novel 3-D based classification approach for HSI applies both spectral and spatial data. It also implements a border imaging policy to efficiently progression border areas in the image, and graphics processing units (GPUs) has been efficiently deployed. The method attained a decrease of the computation time and growing the precision in the classification of HSI than the conventional ANN techniques. The system lacked for multi-resolution. As to address the supervised and semi supervised approaches in classification, neural network based techniques have been proposed in the work carried out before such as (i) Feed forward Neural network (FN) classifiers, used mainly to deal with secondorder optimization-based variations, (ii)Extreme Learning Machine (ELM). Later the concept of CNN was used in multi-hidden-layer networks. The examples of multi hidden layer networks are radial basis function (RBF) networks and Kernel learning networks. ELM-based networks are successfully applied in nonlinear classifier data and showed remarkably efficiency in terms of accuracy and computational complexity. The main three parts of a CNN are a convolutional layer, nonlinear function and a pooling layer. The deep CNN is used to extract the features found in input. The architecture of deep CNN is shown in figure 6 .

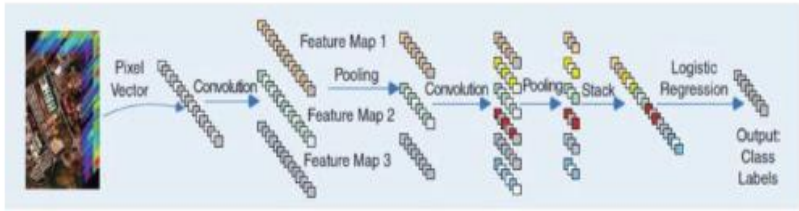

Figure 6: Spectral Classification diagram based on Deep Convolutional Neural Network (Source Intechopen)

CNN was introduced to understand the deep representation of networks. The CNN is based on spectral signatures which are unique in nature. CNN classification approach resulted in a better performance compared to that of SVM. In CNN, during the learning process of unsupervised sparse features levels of spatial features are extracted. In case of deep CNN pixel-pair features were learned to achieve better classification.

DL possesses some drawbacks (i) Over fitting: As to overcome the problem of over fitting (Results are very good on training data sets and not on testing data sets of HSI) in deep learning, powerful regularization methods to be used. (ii) Discriminative representation: This type of problem occurs usually in RS data as they are complex in nature which results in poor feature learning from the objects. (iii) Research in deeper layer of DL: The deeper layers in supervised networks like CNN has complex structural distributions and is the open topic for the researcher to learn.

\section{CHALLENGES AND DISCUSSION}

The main challenges faced by Hyperspectral imaging are curse of high dimensionality, processing time, preprocessing, and atmospheric correction and accessing of the training samples. Some of the challenges are discussed below. The classification efficiency and kappa coefficients obtained using different classification approaches are also discussed.

\section{Case 1:}

Compared to the Contrasted and RBFSVM, the CNN based classifier showed greater classification accuracy where the general data set is considered with all the particular classes as shown in Figure 6. With the expanding time of training, the accuracy of classification of every data in the Hyperspectral can extend over 90\%. In this case, concede that the training procedure is moderately timeexpending to accomplish excellent execution; be that as it may, the proposed CNN classifier has similar points of interest of profound learning algorithms. Besides, our execution of CNN could be upgraded enormously on effectiveness, or it can utilize other CNN structures, for example, Caffe (Jia et al. 2014), to decrease training and test time. As indicated by trials, it takes lesser time to accomplish 90\% accuracy in MNIST dataset (Lecun1998) by utilizing Caffe and it takes over 120 minutes when actual framework is used.

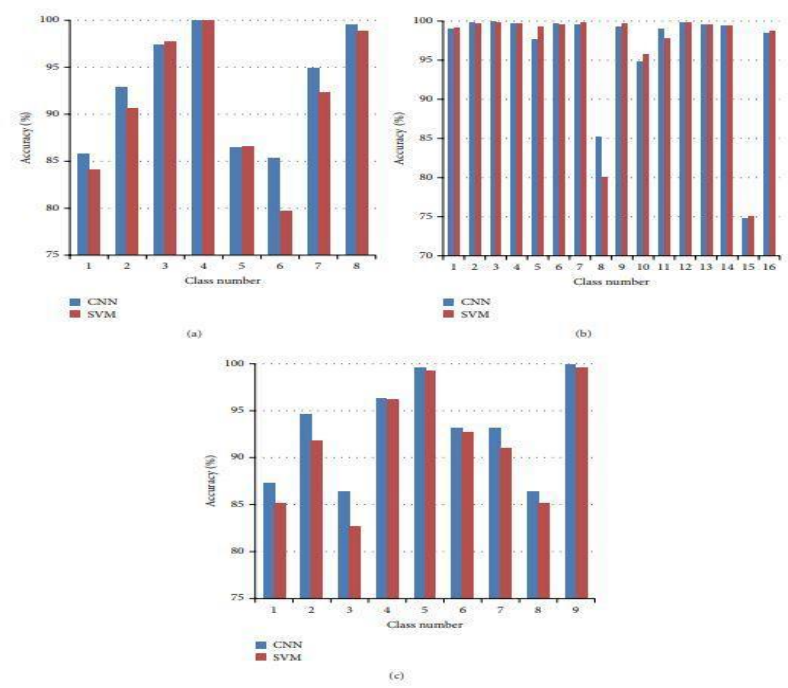

Figure 7: Chart indicating the accuracy in classification for all the classes for different data sets taken. From (a) to (c): Indian Pines, Salinas, and University of Pavia (Hu et al. 2015)

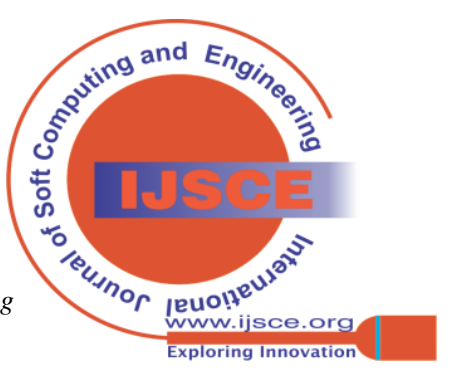




\section{Case 2:}

The below chart indicates that the deepness helps to develop classification accuracy. However, an excessive amount of layers may lower classification outcomes. The quantities of appropriate convolution layers are 5 for Indian Pines, 3 for Pavia, and 4 for KSC data sets.

This is influenced by the dimensionalities increment seen in data sources. The dimensions taken are 200, 103, and 176 for Indian Pines, University of Pavia, and KSC data sets respectively.

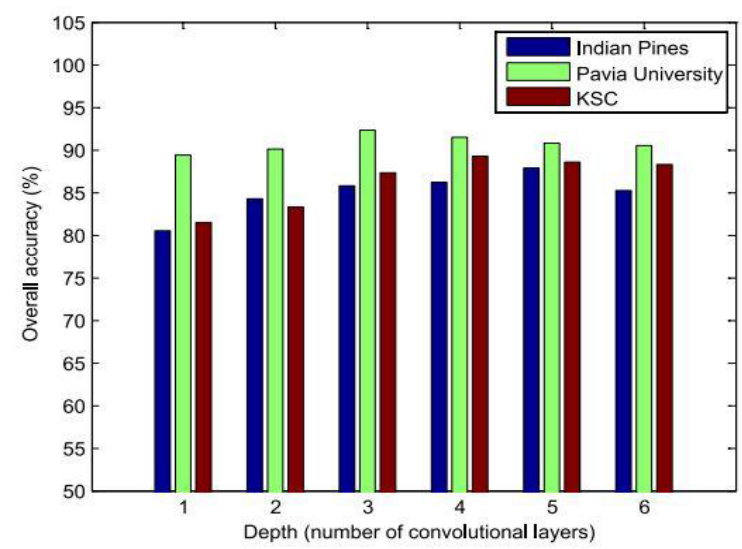

Fig 8: Chart showing the results of CNN based spectral classification for different depths using (Source Chen et al. 2016)

\section{Case 3:}

In this case, it is s recommended to use the SVM technique for classification even if we have small number of datasets of Hyperspectral images, where experimentation with two variations of SVM is experienced. The observed results showed that classification accuracy decayed marginally when the number of features exceeded beyond 50 or so for each of these three classifiers.

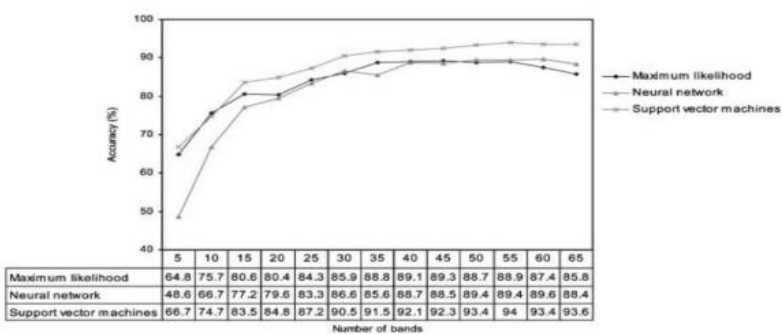

Figure 9: Variation in classification accuracy with developing several features and fixed training dataset

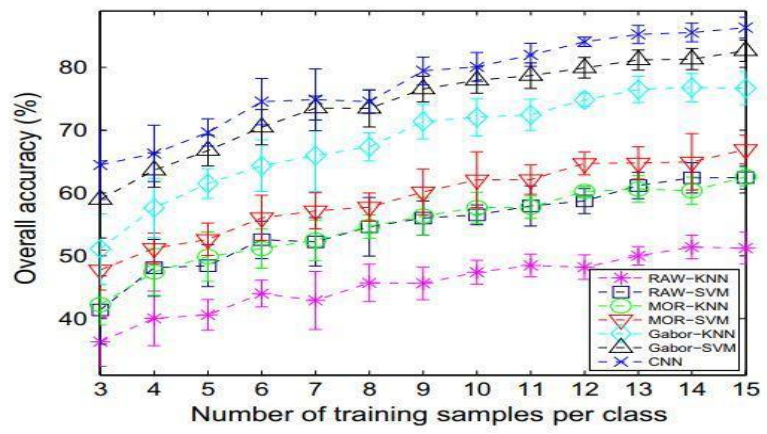

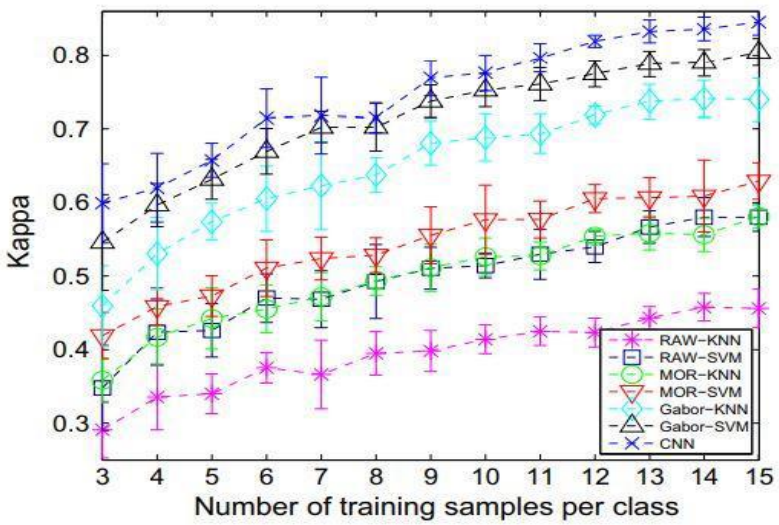

Figure 10: (a) Number of training samples versus overall Accuracy (b) Number of training samples per class Versus kappa values (Source Yu et al. 2017 p.5)

\section{Case 4:}

The case 4, part (a) indicates the variations in the overall accuracy with respect to the number of samples and part (b) shows the variation in kappa coefficient measured as functions of the quantities per class as training samples (3, $4,5 \ldots$ 15). The graph demonstrates that the samples increment in the training dataset has an ideal impact in the exhibition of all the techniques considered. The two classifiers on the raw Hyperspectral data (RAW-KNN and RAW-SVM) convey the most exceeding terrible outcomes as a rule for both the OA and the kappa measures. The exhibition on the Gabor features (Gabor-KNN and Gabor$\mathrm{SVM}$ ) is superior compared to the morphological characteristics (MOR-KNN and MOR-SVM), which is because of the distinguishable intensity of the Gabor features. Further, this CNN strategy reliably gives the best outcomes. Mainly, when the training sample size is less, the contrasts among the algorithms are clearly visible (Yu et al. 2017).

\section{COMPARATIVE STUDY}

The methods, outcomes and the gap in the various surveys done so far are listed in table

\begin{tabular}{|c|l|l|l|l|l|}
\hline $\begin{array}{c}\text { S } \\
\text { N }\end{array}$ & $\begin{array}{l}\text { Autho } \\
\text { r }\end{array}$ & \multicolumn{1}{|c|}{ Method } & Parameter & Outcome & Gap \\
\hline 1 & $\begin{array}{l}\text { Lan et } \\
\text { al. } \\
2019\end{array}$ & $\begin{array}{l}\text { K-sparse de- } \\
\text { noising } \\
\text { autoencoder } \\
\text { (KDAE) and } \\
\text { spectral- } \\
\text { restricted } \\
\text { spatial } \\
\text { characteristi } \\
\text { cs }\end{array}$ & $\begin{array}{l}\text { HSI } \\
\text { classificatio }\end{array}$ & $\begin{array}{l}\text { Existing } \\
\text { defects in } \\
\text { the ranking } \\
\text { of } \\
\text { neighborhoo } \\
\text { d SI are } \\
\text { improved }\end{array}$ & $\begin{array}{l}\text { Improveme } \\
\text { nt in the } \\
\text { SVMs and } \\
\text { DAE } \\
\text { classificatio } \\
\text { nambined } \\
\text { combith } \\
\text { with } \\
\text { developed } \\
\text { features }\end{array}$ \\
\hline
\end{tabular}

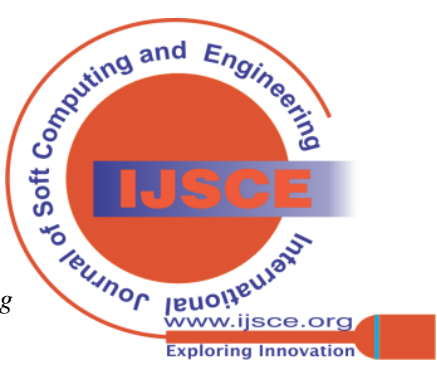


Approaches for Hyperspectral Image Classification- Detailed Review

\begin{tabular}{|c|c|c|c|c|c|}
\hline 2 & $\begin{array}{l}\text { Zhou et al. } \\
2019\end{array}$ & $\begin{array}{l}\text { Spectral- } \\
\text { spatial } \\
\text { LSTMs }\end{array}$ & $\begin{array}{l}\text { HSI } \\
\text { classificat } \\
\text { ion }\end{array}$ & $\begin{array}{l}\text { Achieves } \\
\text { better } \\
\text { performanc } \\
\text { e of } \\
\text { classificati } \\
\text { on in terms } \\
\text { of Kappa } \\
\text { coefficient } \\
\text { and overall } \\
\text { accuracy. } \\
\text { Better } \\
\text { performanc } \\
\text { e than } \\
\text { other } \\
\text { methods }\end{array}$ & $\begin{array}{l}\text { Further } \\
\text { improveme } \\
\text { nt can be } \\
\text { made in the } \\
\text { results } \\
\text { obtained } \\
\text { for } \\
\text { classificati } \\
\text { on using } \\
\text { different } \\
\text { methods on } \\
\text { the KSC } \\
\text { dataset }\end{array}$ \\
\hline 3 & $\begin{array}{l}\text { Medjahed\& } \\
\text { Ouali } 2018\end{array}$ & $\begin{array}{l}\text { Stochastic } \\
\text { method }\end{array}$ & $\begin{array}{l}\text { HSI } \\
\text { classificat } \\
\text { ion }\end{array}$ & $\begin{array}{l}\text { Reduction } \\
\text { in the } \\
\text { classificati } \\
\text { on error } \\
\text { rate }\end{array}$ & $\begin{array}{l}\text { Classificati } \\
\text { on } \\
\text { accuracy } \\
\text { rate } \\
\text { depends } \\
\text { largely on } \\
\text { the selected } \\
\text { features }\end{array}$ \\
\hline 4 & $\begin{array}{l}\text { Pan et al. } \\
2018\end{array}$ & $\begin{array}{l}\text { Collaborat } \\
\text { ive } \\
\text { learning } \\
\text { framewor } \\
\mathrm{k}\end{array}$ & $\begin{array}{l}\text { HSI } \\
\text { classificat } \\
\text { ion }\end{array}$ & $\begin{array}{l}\text { CLUC can } \\
\text { be more } \\
\text { efficiency } \\
\text { in time } \\
\text { consumpti } \\
\text { on }\end{array}$ & $\begin{array}{l}\text { Methods } \\
\text { like SVM, } \\
\text { MCLU can } \\
\text { be a } \\
\text { research } \\
\text { topic in } \\
\text { future work }\end{array}$ \\
\hline 5 & $\begin{array}{l}\text { Zhao et al. } \\
2018\end{array}$ & $\begin{array}{l}\text { SODFN } \\
\text { and } \\
\text { FCLFN }\end{array}$ & $\begin{array}{l}\text { HSI } \\
\text { classificat } \\
\text { ion }\end{array}$ & $\begin{array}{l}\text { This } \\
\text { approaches } \\
\text { steadily } \\
\text { demonstrat } \\
\text { e healthier } \\
\text { performanc } \\
\text { e than the } \\
\text { other } \\
\text { methods }\end{array}$ & $\begin{array}{l}\text { Train a } \\
\text { deep CNN } \\
\text { with great } \\
\text { generalizati } \\
\text { on ability } \\
\text { in the } \\
\text { future } \\
\text { works }\end{array}$ \\
\hline 6 & $\begin{array}{l}\text { Peng et al. } \\
2018\end{array}$ & $\begin{array}{l}\text { LAJSR } \\
\text { and JSR } \\
\text { method }\end{array}$ & $\begin{array}{l}\text { Hyperspe } \\
\text { ctral } \\
\text { remote } \\
\text { sensing } \\
\text { images }\end{array}$ & $\begin{array}{l}\text { LAJSR } \\
\text { technique } \\
\text { is highly } \\
\text { effective } \\
\text { than } \\
\text { existing } \\
\text { JSR and } \\
\text { SVM }\end{array}$ & $\begin{array}{l}\text { Implement } \\
\text { ation of } \\
\text { CNN in the } \\
\text { proposed } \\
\text { algorithm } \\
\text { will be } \\
\text { more } \\
\text { effective in } \\
\text { getting } \\
\text { better } \\
\text { classificati } \\
\text { on results } \\
\text { when } \\
\text { compared } \\
\text { with other } \\
\text { algorithm }\end{array}$ \\
\hline 7 & $\begin{array}{l}\text { Zhang et al. } \\
2018\end{array}$ & $\begin{array}{l}\text { Augmente } \\
\text { d } \\
\text { Lagrangia } \\
\text { n Method }\end{array}$ & $\begin{array}{l}\text { Robust } \\
\text { manifold } \\
\text { matrix } \\
\text { factorizati } \\
\text { on }\end{array}$ & $\begin{array}{l}\text { Convergen } \\
\text { ce could be } \\
\text { arrived in } \\
\text { less than } \\
\text { ten } \\
\text { iterations. }\end{array}$ & $\begin{array}{l}\text { An } \\
\text { additional } \\
\text { out-of- } \\
\text { sample } \\
\text { extension } \\
\text { trick is } \\
\text { suggested } \\
\text { to make the } \\
\text { method can } \\
\text { deal with } \\
\text { the large- } \\
\text { scale HSI }\end{array}$ \\
\hline 8 & $\begin{array}{l}\text { Liu et al. } \\
2018\end{array}$ & $\begin{array}{l}\text { Fuzzy } \\
\text { rough set } \\
\text { theory }\end{array}$ & $\begin{array}{l}\text { Soybean } \\
\text { classificat } \\
\text { ion }\end{array}$ & $\begin{array}{l}\text { IM-FRS } \\
\text { based band } \\
\text { selection } \\
\text { algorithm } \\
\text { can } \\
\text { provide } \\
\text { stable } \\
\text { results }\end{array}$ & $\begin{array}{l}\text { According } \\
\text { to need, the } \\
\text { parameters } \\
\text { can be set } \\
\text { flexibly }\end{array}$ \\
\hline 9 & $\begin{array}{l}\text { Qureshi et } \\
\text { al. } 2018\end{array}$ & $\begin{array}{l}\text { Hyperspe } \\
\text { ctral } \\
\text { Imaging } \\
\text { systems }\end{array}$ & $\begin{array}{l}\text { Challenge } \\
\mathrm{s} \text { in } \\
\text { acquiring } \\
\text { and pre- } \\
\text { processin }\end{array}$ & $\begin{array}{l}\text { Pattern } \\
\text { recognition } \\
\text { technique } \\
\text { was } \\
\text { introduced }\end{array}$ & $\begin{array}{l}\text { HSI can be } \\
\text { implement } \\
\text { ed on } \\
\text { upcoming } \\
\text { application }\end{array}$ \\
\hline
\end{tabular}

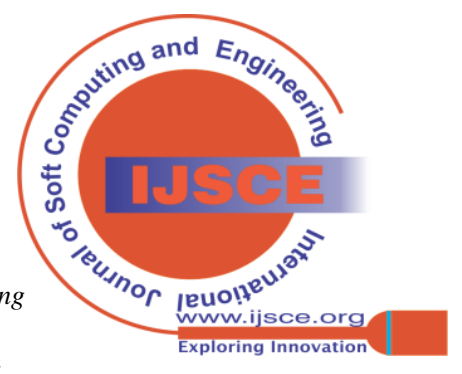


Table 1: Results of Comparison between D-CNN and SVM classifiers for 2 data sets

\begin{tabular}{|l|l|l|}
\hline Data set & D-CNN & SVM \\
\hline University of Pavia & $92.64 \%$ & $90.42 \%$ \\
\hline Indian Pines & $90.18 \%$ & $82.54 \%$ \\
\hline
\end{tabular}
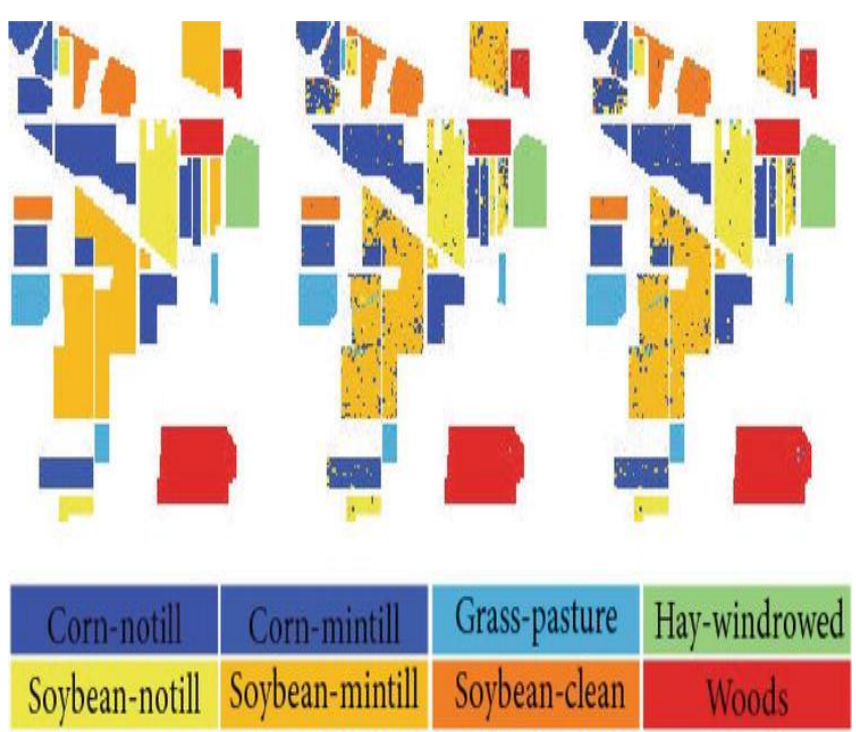

Figure 11: RGB composition maps obtained as a result of classification for the Indian Pines data set. From left to right: Ground truth, SVM classification, and D-CNN approach (Source Intechopen)

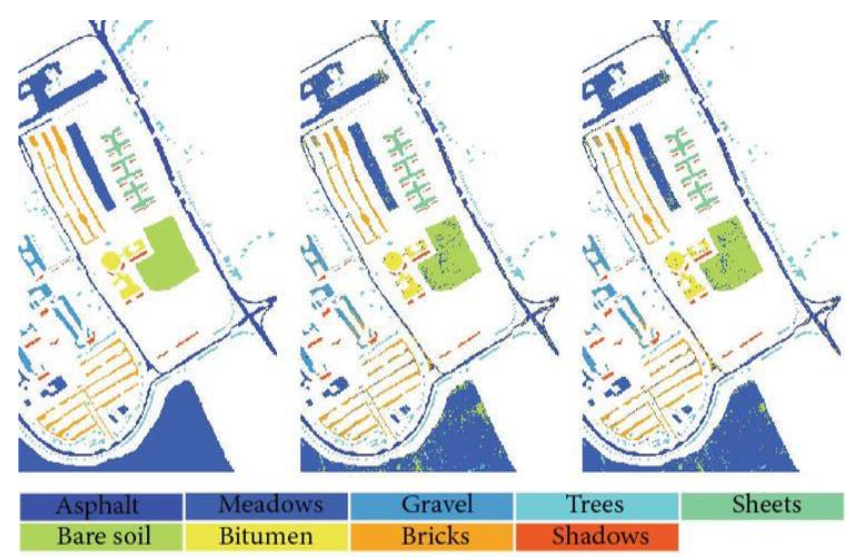

Figure 12: Composition maps resulting from the result of classification obtained using the datasets of University of Pavia. From left to right: Ground truth, SVM classification and D-CNN approach (Source Intechopen)

\section{CONCLUSION}

In these survey different approaches used in Hyperspectral image classification is discussed. The variations in accuracy performance, overall accuracy and Kappa coefficients for different HSI classification techniques are listed. The limitations of accurate data and possible solutions for obtaining more accuracy are listed. The investigation surveyed here has recognized that the neural network method is viable for the HSI classification. Similarly, performance of that conventional classifier, such as CNN, KNN, ANN, and SVM has been attained. However further work is essential before the neural network becomes a feasible alternative to HSI classifier. This paper has analysed partially with collection of previous records on HSI performance accuracy using neural network. From the different case studies we could conclude that a new CNNbased approach for classification of HSI in comparison with SVM and DNN-based classifier gives high accuracy even if the training samples were less. The survey may motivate researchers in developing new techniques with performance efficiency in the context of HSI using neural network. The deep learning technology can be made as a hybrid technology by combining with other existing methods like sparse coding, ensemble coding. The hybrid method can be a future research area in Hyperspectral Image Classification.

\section{REFERENCES}

1. Rahul Nigam1, Rojalin Tripathy, Sujay Dutta, Nita Bhagia, Rohit Nagori, K. Chandrasekar, Rajsi Kot, Bimal K. Bhattacharya and Susan Ustin (2019) "Crop type discrimination and health assessment using Hyperspectral imaging" Journal of Current Science, Vol. 116, 10.

2. Qureshi, R., Uzair, M., Khurshid, K., \& Yan, H. (2019), "Hyperspectral document image processing: Applications, challenges, and future prospects. Pattern Recognition", 90, 12-22.

3. Zhang, L., Zhang, L., Du, B., You, J., \& Tao, D. (2019), "Hyperspectral Image Unsupervised Classification by Robust Manifold Matrix Factorization", Information Sciences, 485, 154-169.

4. Liu, Y., Wu, T., Yang, J., Tan, K., \& Wang, S (2019), "Hyperspectral band selection for soybean classification based on information measure in FRS theory, Bio- systems Engineering, 2019 178, 219-232.

5. Zhou, F., Hang, R., Liu, Q., \& Yuan, X. (2019), "Hyperspectral image classification using spectral-spatial LSTMs", Neurocomputing, 328, 39 $-47$.

6. Lan, R., Li, Z., Liu, Z., Gu, T., \& Luo, X. (2019), "Hyperspectral image classification using k-sparse de- noising autoencoder and spectral-restricted spatial characteristics", Applied Soft Computing, 2019, pp 74, 693-708.

7. Carlan, I, Mihai, B. A., Nistor, C., \& Grobe-Stoltenberg (2019), "Identifying urban vegetation stress factors based on open access remote sensing imagery and field observations", Ecological Informatics 55, pp. 01032.

8. Hasan, M, Ullah, S., Khan, M. J., \& Khurshid, K (2019), “Comparative Analysis of SVM, ANN and CNN for Classifying Vegetation Species Using Hyperspectral Thermal Infrared Data”, International Archives of the Photogrammetric, Remote Sensing \& Spatial Information Sciences.

9. Sun, Z., Zhao, X., Wu, M. and Wang, C (2019), "Extracting Urban Impervious Surface from WorldView-2 and Airborne LiDAR Data Using 3D Convolutional Neural Networks", Journal of the Indian Society of Remote Sensing, 47(3), pp.401-412.

10. Brabant Ca, Alvarez-Vanhard E., Morin G., Thanh Nguyen K., Laribi A., Houet T (2018), "Evaluation of Dimensional Reduction Methods on Urban Vegetation Classification Performance using hyperspectral Data”, IEEE, pp.1636-1639.

11. Cao Jinging, Wanchun Leng, Kai Liu, Lin Liu, Zhi He ID and Yuanhui Zhu (2018), "Object-Based Mangrove Species Classification Using Unmanned Aerial Vehicle Hyperspectral Images and Digital Surface Models", Article published in Hyper Spectral Remote Sensing for Forest and Trees outside Forests.

12. Peng, J, Jiang, X., Chen, N., \& Fu, H (2018), "Local Adaptive Joint Sparse representation for Hyperspectral image classification. Neuro computing", 2019 334, 239-248.

13. Zhao, G., Liu, G., Fang, L., Tu, B., \& Ghamisi, P (2018), Multiple Convolutional Layers Fusion Framework for Hyperspectral Image Classification. Neurocomputing, 2019, pp 339, 149-160.

14. Yinhua, X Gao, R Wei - Optik (2018), "Hyper spectral image classification based on adaptive segmentation", Optik Volume 172.

15. Annala, L., Eskelinen, M., Hämäläinen, J., Riihinen, A. and Pölönen, I (2018), "Practical approach for Hyperspectral image processing in python", International Archives of the Photogrammetry, Remote Sensing and Spatial Information Sciences (Vol. 42, No.3), International Society for Photogrammetry and Remote Sensing.

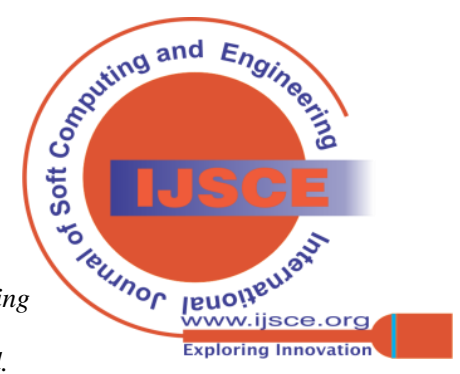




\section{Approaches for Hyperspectral Image Classification- Detailed Review}

16. Brabant, C., Alvarez-Vanhard, E., Morin, G., Laribi, A. and Houet, T (2018), "Evaluation of Dimensional Reduction Methods on Urban Vegetation Classification Performance Using Hyperspectral Data", IGARSS 2018-2018 IEEE International Geosciences and Remote Sensing Symposium (pp. 1636-1639), IEEE.

17. Ouerghemmi, W., Gadal, S. and Mozgeris (2018), "Urban Vegetation Mapping using Hyperspectral Imagery and Spectral Library", IGARSS 2018-2018 IEEE International Geosciences and Remote Sensing Symposium (pp. 1632-1635), IEEE.

18. Ishida, T., Kurihara, J., Viray, F.A., Namuco, S.B., Paringit, E.C., Perez, G.J., Takahashi, Y. and Marciano Jr, J.J (2018), "A Novel Approach for Vegetation Classification using UAV-based Hyperspectral Imaging”, Computers and electronics in agriculture, 144, pp.80-85.

19. Audebert, N., Le Saux, B. and Lefèvre, S (2018), "Beyond RGB: Very high resolution urban remote sensing with multimodal deep networks", ISPRS Journal of Photogrammetry and Remote Sensing, 140, pp.20-32.

20. Binge Cui et al (2017), "A noval Hyper spectral image classification approach based on multiresolution segmentation with a few labeled samples", Binge Cui et al, International Journal of Advanced Robotic Systems.

21. Namita M Kulkarni (2017), "Crop Identification Using Un-supervised ISODATA and K-Means from Multispectral Remote Sensing Imagery" Int. Journal of Engineering Research and Application ISSN: 22489622, Vol. 7, Issue 4, (Part -1), and pp.45.

22. R.Hang, Q. Liu, H. Song, Y. Sun, F. Zhu, H. Pei (2017) , "Graph regularized nonlinear ridge regression for remote sensing data analysis, IEEE Journal of Selected Topics in Applied Earth Observations and Remote Sensing”, 10 (1) 277-285.

23. Zelang Miao, Wezhong Shi (2016), "A New Methodology for SpectralSpatial Classification of Hyperspectral Images", Journal of Sensors, Article ID 1538973.

24. Dian, Y., Pang, Y., Dong, Y., \& Li, Z. (2016), "Urban tree species mapping using airborne LiDAR and Hyperspectral data", Journal of the Indian Society of Remote Sensing, 44(4), 595-603.

25. Feng, Q, Liu, J. and Gong, J (2015), "UAV remote sensing for urban vegetation mapping using random forest and texture analysis", Remote sensing, 7(1), pp.1074-1094.

26. Buyadi, S.N.A., Mohd, W.M.N.W. and Misni, A (2015), "Vegetation's Role on Modifying Microclimate of Urban Resident", Procedia - Social and Behavioral Sciences, 202, pp.400-407.

27. Rajesh K. Dhumal, Amol D. Vibhute, Ajay D. Nagne, Yogesh D Rajendra, Karbhari V. Kale and Suresh C. Mehrotra (2015), "Advances in Classification of Crops using Remote Sensing Data", International Journal of Advanced Remote Sensing and GIS.

28. R. N. Sahoo1, S. S. Ray2 and K. R. Manjunath (2015), "Hyperspectral remote sensing of agriculture" Current Science, Vol. 108, No. 5, 10.

29. M. Arafat, Mohamed A. Aboelghar, Eslam F. Ahmed (2013), "Crop Discrimination Using Field Hyper Spectral Remotely Sensed Data" Advances in Remote Sensing, 2, pp. 63-70.

30. Tigges, J., Lakes, T. and Hostert, P (2013), "Urban vegetation classification: Benefits of multi temporal Rapid Eye satellite data. Remote Sensing of Environment", 136, pp 66-75.

31. Zhang, C. and Xie, Z (2013), "Combining object-based texture measures with a neural network for vegetation mapping in the Everglades from Hyperspectral imagery", Remote Sensing of Environment, 124, pp.310-320.

32. Ramdani, F (2013), "Urban vegetation mapping from fused Hyperspectral Image and LiDAR data with Application to Monitor Urban Tree Heights", Journal of Geographic Information System, 5(04), pp.404

\section{AUTHORS PROFILE}

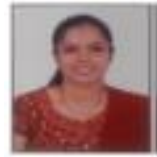

Kushalatha.M.R, received Bachelor's degree and Master's degree from VTU, Belgaum and currently research scholar under VTU. She is working as an Assistant Professor in the Department of Electronics and communication Engineering, Nitte Meenakshi Institute of Technology, Bangalore and has a teaching experience of 13 years. Her area of research is "Hyperspectral imaging" and Signal processing.

Dr. Prasantha.H.S. received Bachelor's degree from Bangalore University, Master's Degree from VTU Belgaum and $\mathrm{PhD}$ from Anna University, Chennai, in the area of signal and image processing. He has 20+ years of teaching and research experience. His research interests includes Multimedia and Signal Processing. He has published more than 35 papers in International conferences and Journals. He is the reviewer for various reputed conferences and Journals. He is currently guiding four students for their research program under VTU. .Currently, he is working as a Professor in the department of Electronics and Communication Engineering, Nitte Meenakshi Institute of Technology (Affiliated to VTU, Belgaum), Bangalore

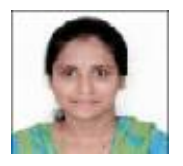

Beena.R.Shetty, is currently working as an Assistant Professor in the department of Electronics and Communication in Nitte Meenakshi Institute of Technology, Bangalore has a total teaching experience of 8 years. Her areas of research are Image processing
Published By:

Blue Eyes Intelligence Engineering and Sciences Publication (C) Copyright: All rights reserved.

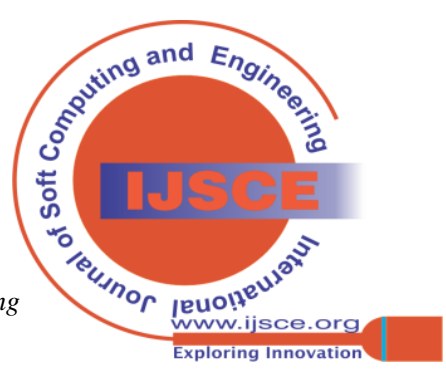

Exploring Innovation 